\title{
Right-sided endomyocardial fibrosis with recurrent pulmonary emboli leading to irreversible pulmonary hypertension
}

\begin{abstract}
A 26 year old Saudi man with features of both Loeffer's endocarditis and endomyocardial fibrosis presented with mild symptoms and pulmonary emboli. Echocardiographic examination showed obliteration of the right ventricular apex by an attached mass. The results of haemodynamic studies were somewhat abnormal and medical treatment was started. Despite anticoagulation with warfarin the patient's condition deteriorated rapidly over a four month period after a further episode of pulmonary embolism and the development of pulmonary hypertension. Two haemodynamic studies performed four months apart were typical of pulmonary hypertension and later right ventricular failure; they showed none of the characteristics of restriction. Pulmonary embolectomy was attempted but there was no cleavage plane between the organised thrombi and the endothelium of the pulmonary artery. The patient died of severe pulmonary hypertension and right ventricular failure several days after operation.

Surgical intervention in the early stages of right-sided endomyocardial fibrosis might have prevented the development of pulmonary embolism and pulmonary hypertension.
\end{abstract}

\section{(Br Heart J 1992;68:326-9)}

Loeffer's endocarditis and endomyocardial fibrosis were long regarded as separate entities. Now Loeffer's endocarditis and endomyocardial fibrosis are generally regarded as manifestations of the same disease. ${ }^{1-3}$ The pathological findings in advanced disease are identical for both conditions. ${ }^{1-4}$ We describe a patient who had features of both Loeffer's endocarditis and endomyocardial fibrosis.

This patient presented at a relatively early stage of the disease when the right ventricle only was affected. At the time the patient had sustained a small pulmonary emboli and the haemodynamic function was somewhat abnormal. Despite anticoagulation with warfarin there were further episodes of pulmonary embolism and pulmonary hypertension and severe right heart failure developed. The surgical and pathological findings are described.
Case report

A 26 year old Saudi man living in Gizan was referred from the Riyadh Central Hospital for cardiac catheterisation. He had been complaining of dyspnoea and chest pain on exertion for the past six weeks. He was admitted after an episode of dizziness and chest pain at rest. The patient had had bronchial asthma from the age of five to the age of 16 . He smoked 30 cigarettes a day. He had a rash eight years ago and a further two episodes of rash during the past two years. In November 1989 the jugular venous pulse was normal and the carotid upstrokes were normal. The chest was clear and blood pressure was $104 / 80 \mathrm{~mm} \mathrm{Hg}$. The pulse was 70 per minute and regular. The left ventricular impulse was normal and at auscultation a physiological split second heart sound was heard. P2 was normal. A pansystolic murmur (grade 1/4) was detected at the left sternal border. The liver was not palpable. There was no radiofemoral delay and all peripheral pulses were present with no bruits. There were no genital or mouth ulcers.

Echocardiographic studies showed obliteration of the right ventricular apex by a mass in the right ventricular cavity attached by a pedical (fig 1A). The patient was treated with intravenous heparin and was transferred to our hospital for further cardiac investigations. The full blood count was normal with $2 \%$ of eosinophils. The renal and liver profiles were normal. Protein electrophorisis showed a slight diffuse increase in $\gamma$ globulins and the erythrocyte sedimentation was $11 \mathrm{~mm} / \mathrm{h}$. Protein $\mathrm{C}$ was $0.59 \mathrm{U} / \mathrm{ml}$ (normal between 0.64 and $1 \cdot 13$ ) and protein $S$ was $0.37 \mathrm{U} / \mathrm{ml}$ (normal $0.51-0.91)$-low and consistent with oral anticoagulation. Serological tests for schistosomiasis were negative. Antithrombin III was $90 \mathrm{mg} / \mathrm{ml}$ (normal $=80-120$ ) and plasminogen 118 (normal $=80-120 \%$ ). The partical thromboplastin time was 63 seconds while he was being given intravenous heparin. The chest $x$ ray showed a heart of normal size and clear lung fields. The electrocardiogram showed sinus rhythm and incomplete right bundle branch block and non-specific ST-T changes. Holter monitoring showed periods of 2:1 atrioventricular block and a few ventricular extrasystoles. Echocardiographic studies showed a left ventricle of normal size and function. The right atrium was dilated and an irregular mass obliterated the right ventricular apex and extended to half way up the ven- 

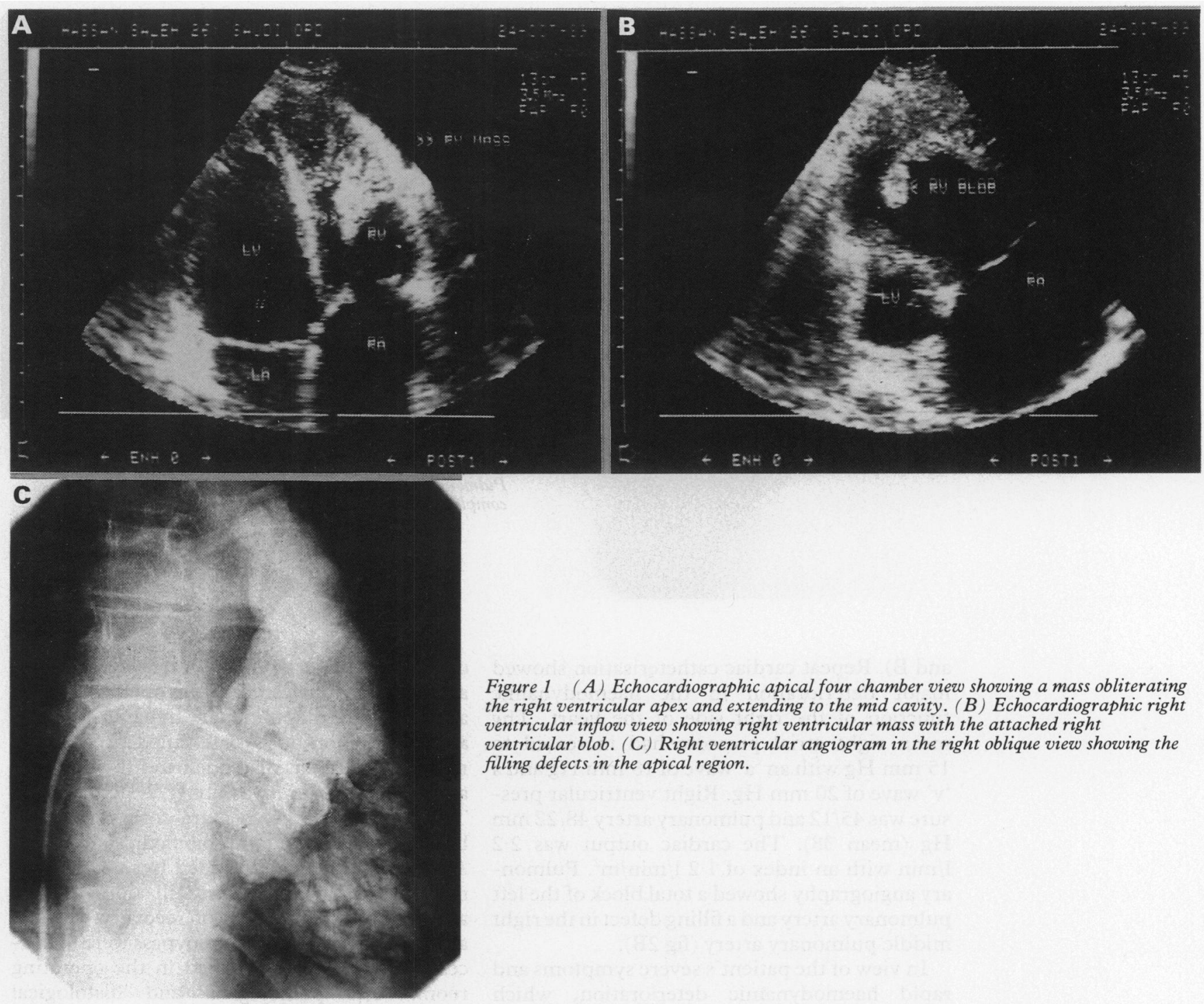

Figure 1 (A) Echocardiographic apical four chamber view showing a mass obliterating the right ventricular apex and extending to the mid cavity. (B) Echocardiographic right ventricular inflow view showing right ventricular mass with the attached right ventricular blob. (C) Right ventricular angiogram in the right oblique view showing the filling defects in the apical region.

tricular cavity (figs 1 and 2). Colour Doppler showed moderate tricuspid regurgitation. Cardiac catheterisation showed no haemodynamic evidence of constrictive or restrictive physiology or both. The mean right atrial pressure was $8 \mathrm{~mm} \mathrm{Hg}$ with an 'a' wave of 13 and a ' $v$ ' wave of $10 \mathrm{~mm} \mathrm{Hg}$. The right ventricular pressure was $33 / 3 \mathrm{~mm} \mathrm{Hg}$ and the pulmonary artery pressure was $33 / 19$ (mean 24 ) $\mathrm{mm} \mathrm{Hg}$. Pulmonary capillary wedge was $7 \mathrm{~mm} \mathrm{Hg}$ and left ventricular pressure 104/ $12 \mathrm{~mm} \mathrm{Hg}$. The left ventricular angiogram was normal. The right ventriculogram showed moderate tricuspid regurgitation and a dilated right ventricular cavity. There were filling defects in the right ventricular apex (not a typical feature of endomyocardial fibrosis) without evidence of right ventricular apex cut off (fig 1C). The pulmonary angiogram showed occlusion of the pulmonary arteries supplying the left, mid and lower lobes (fig 2A). A biopsy specimen of the right ventricle taken in November 1989 showed a thrombus containing degranulated eosinophils with minimal myocardial fibrosis. At this stage the diagnosis was early stage endomyocardial fibrosis with pulmonary emboli. Because the symptoms were mild and cardiac haemodynamics function virtually normal we decided to continue anticoagulation treatment with warfarin. Beçhet's disease was excluded and the cardiac biopsy specimen, though not pathognomonic, indicated endomyocardial fibrosis. The patient was followed up in the outpatient clinic and warfarin anticoagulation was regularly monitored (prothrombin time ratio of $2: 1$ ).

In January 1990 the patient was reviewed in the outpatient clinic and was still doing well and the echocardiographic study showed evidence of a right ventricular mass (figs 1 and 2). From then onwards there was a gradual clinical deterioration and in March 1990 the patient was unwell. During the past month he deteriorated considerably and was now in New York Heart Association Class IV with shortness of breath on minimal exertion and orthopnoea. When the patient was admitted the jugular venous pulse was raised to the angle of the jaw. The heart rate was 85 beats $/ \mathrm{min}$ and regular. There was a pansystolic murmur grade $3 / 4$ at the sternal border and a striking gallop rhythm. The liver was palpable $4 \mathrm{~cm}$ below the costal grade. There was ascites. The electrocardiogram showed right axis deviation and evidence of right ventricular hypertrophy and right bundle branch block. Echocardiographic studies showed a persistent right ventricular mass and severe tricuspid regurgitation (figs $1 \mathrm{~A}$ 

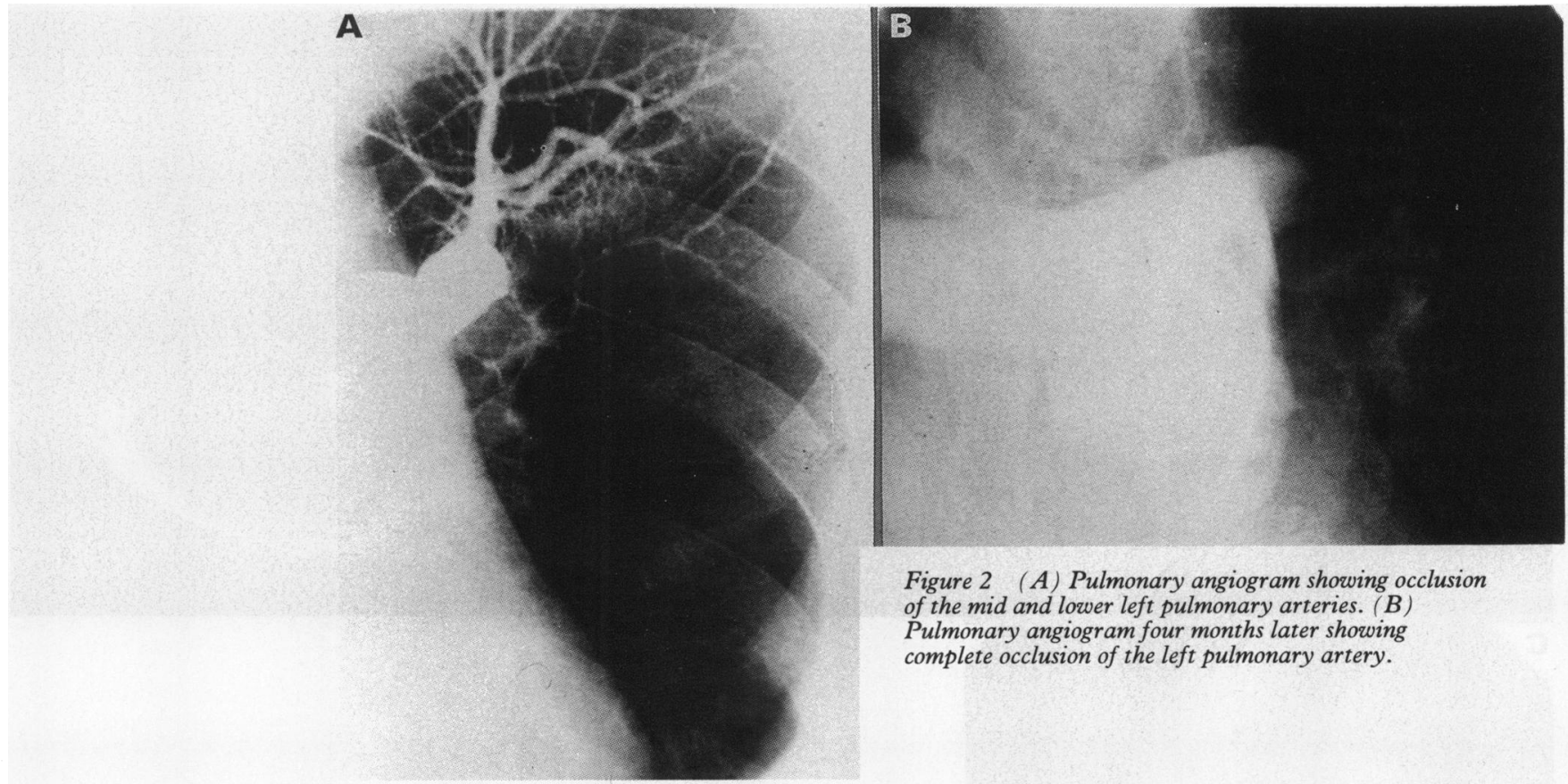

and B). Repeat cardiac catheterisation showed major deterioration in the haemodynamic function of the right side of the heart. The mean right atrial pressure had increased to $15 \mathrm{~mm} \mathrm{Hg}$ with an 'a' wave of $18 \mathrm{~mm} \mathrm{Hg}$ and a ' $v$ ' wave of $20 \mathrm{~mm} \mathrm{Hg}$. Right ventricular pressure was $45 / 12$ and pulmonary artery $48 / 22 \mathrm{~mm}$ $\mathrm{Hg}$ (mean 38). The cardiac output was $2 \cdot 2$ $1 / \mathrm{min}$ with an index of $1.2 \mathrm{l} / \mathrm{min} / \mathrm{m}^{2}$. Pulmonary angiography showed a total block of the left pulmonary artery and a filling defect in the right middle pulmonary artery (fig $2 \mathrm{~B}$ ).

In view of the patient's severe symptoms and rapid haemodynamic deterioration, which were caused by further pulmonary emboli, we decided to attempt to excise the right ventricular mass and perform a pulmonary embolectomy. At operation the posterior and septal leflets of the tricuspid valve were tethered by the fibrous tissue that was a continuation of the papillary muscle. The apical and septal mass was seen in the right ventricle. The mass was whitish and fibrous strands penetrated into the myocardium, which was ulcerated and covered in thrombus. The mass extended to the outflow tract of the right ventricle. The left pulmonary artery was occluded with a recent thrombus, superimposed on old fibrous whitish material that was completely attached to the endothelium of the pulmonary artery. There was no cleavage plane.

The right ventricular mass was excised and the tricuspid valve was replaced by a Hancock 33 bioprosthesis. Embolectomy was impossible because the whitish material of the pulmonary artery was attached to the endothelium. Left pneumectomy was performed because there was considerable bleeding in the endotracheal tube during weaning of bypass. The patient was transferred to the intensive care unit in a reasonable condition. After being extubated on the third postoperative day respiratory acidosis developed and the patient required reintubation. He sustained a cardiac arrest that required

open chest massage in the intensive care unit and he was transferred to the operating room and put on cardiopulmonary bypass. Attempts at weaning him off bypass failed because the right ventricle was distended and the pulmonary artery pressure was above systemic level. The patient was given extra-corporeal membrane oxygenation and biomedical support. After 10 hours of right-sided bypass the pulmonary artery pressure was still suprasystemic and there were no signs of recovery. Several attempts at weaning him off bypass were unsuccessful and the patient died in the operating room. The pathological and histological examination of the specimens of the right ventricle and left pulmonary artery showed features that were characteristic of endomyocardial fibrosis with superimposed thrombus.

\section{Discussion}

Our patient had features that were typical of both Loeffer's endocarditis and endomyocardial fibrosis. ${ }^{5-9}$ His history of asthma, rash, multiple pulmonary emboli, is characteristic of Loeffer's disease. The absence of peripheral eosinophils, isolated right ventricular disease, and absence of systemic illness are features of endomyocardial fibrosis. Although endomyocardial fibrosis and Loeffer's disease used to be regarded as separate clinical entities there is a growing consensus that they are different stages of the same disease. ${ }^{1-4}$ Our patient was probably in an intermediate stage of the diseases.

The right ventricle alone is affected in $10 \%$ of cases of endomyocardial fibrosis. ${ }^{10}$ The restrictive physiology is the haemodynamic hallmark of the disease. ${ }^{10}$ Our patient, however, presented with normal haemodynamic function and without the features of restriction, despite the large right ventricular mass detected on echocardiography. Despite appropriate anti- 
coagulation the disease progressed rapidly with further pulmonary emboli and pulmonary hypertension with right ventricular failure developed. This contrasts with the usual pattern when right heart failure is caused by restriction of the right ventricle. ${ }^{10}$

The course in our patient suggests that earlier excision of the right ventricular mass might have prevented further pulmonary emboli. Medical treatment with anticoagulation did not prevent the progression of the disease to fatal pulmonary embolism. The initial angiogram was misleading because it did not show the classic ventricular apex cut off of endomyocardial fibrosis. When we reviewed the angiogram we found some filling defects that others have described as early evidence of endomyocardial fibrosis. ${ }^{11}$ Nevertheless, the echocardiographic appearance at the time showed a large apical mass extending to the middle of the right ventricle cavity. Colour Doppler examination showed some blood flow to the right ventricular apex in between the mass and this accounted for the angiographic appearance. The nuclear magnetic resonance examination accorded with echocardiographic findings.

We conclude that early surgical intervention may be indicated in the early stages of endomyocardial fibrosis/Loeffer's disease with right-sided involvement to prevent pulmonary emboli and the development of pulmonary hypertension.

1 Roberts WC, Buja LM, Ferran VJ. Loeffler's fibroplastic parietal endocarditis, eosinophilic leukemia, a Davies endomyocardial fibrosis: The same disease at differen states. Pathol Microbiol 1970;35:90-5.

2 Davies J, Spry CJF, Viyaya Raghaven G, Desousa JA. A comparison of the clinical and cardiological features of endomyocardial disease in temperate and tropical regions. Postgrad Med J 1983;59:179-86.

3 Olsen EG. Pathological aspects of endomyocardial fibrosis. Postgrad Med J 1983;59:135-42.

4 Roberts WC, Liegeler DG, Carbone PP. Endomyocardial disease and eosinophilic. A clinical and pathologic spectrum. Am J Med 1969;48:28-42.

5 Spry CJ. The hypereosinophilic syndrome. Clinical features, laboratory findings and treatment. Allergy 1982 37:539-46.

6 Fauci AS, Harley JB, Roberts WC, Ferrans VJ, Gralnick $\mathrm{HR}$, Biornson BH. NIH conference. The idiopathic hypereosinophilic syndrome. Clinical, pathophysiologic hypereosinophilic syndrome. Clinical, pathophysiologic
and therapeutic considerations. Ann Intern Med 1982; and therape

7 Davies JNP, Coles RM. Some considerations regarding obscure disease affecting the normal endocardium. $\mathrm{Am}$ obscure disease affecting

8 Cherian KM, John TA, Abraham KA. Endomyocardial fibrosis clinical profile and role of surgery in management. Am Heart J 1983;105:706-10.

9 Fawzy ME, Halim M, Ziady G, Guindy R, Mercer EN, Feteih N. Endomyocardial fibrosis: Report of eight cases. $J$ Am Coll Cardiol 1985;5:983-89.

10 Cardiomyopathies. Geneva World Health Organization 1984.

11 Sasidharan K, Kartha CC, Balkrishnan KG, Valiathan MJ. Early angiographic features of right ventricular endomyocardial fibrosis. Cardiology 1985;70:127-31. 